\title{
金属有机框架分离纯化 $\mathrm{C}_{4} \sim \mathrm{C}_{6}$ 碳氢化合物的研究
}

\author{
郭振涁 ${ }^{a}$ 张媛媛 ${ }^{*} b$ 冯霄 ${ }^{a}$ \\ $\left({ }^{a}\right.$ 北京理工大学化学与化工学院 北京 100081) \\ $\left({ }^{b}\right.$ 北京理工大学前沿交叉科学研究院 北京 100081)
}

\begin{abstract}
摘要 $\mathrm{C}_{4} \sim \mathrm{C}_{6}$ 碳氢化合物作为重要的化工原料和能源, 在传统的石化工业生产中, 主要通过精馏进行分离提纯, 此过 程能耗高、设备费用昂贵、经济效益低. 利用固体吸附剂进行吸附式分离, 不仅可以降低能源成本, 而且可以提高效率. 金属有机框架(metal-organic frameworks, MOFs) 作为一类由金属离子或团簇和有机单体组装而成的晶态多孔材料, 具 有高孔隙率、规整开放的孔道、丰富的官能团和多样的结构, 在气体储存与分离中有着良好的应用前景. 介绍了 $\mathrm{C}_{4} \sim$ $\mathrm{C}_{6}$ 碳氢化合物分离的重要性, 并从 MOFs 分离机制出发, 概述了目前 MOF 材料用于分离纯化 $\mathrm{C}_{4} \sim \mathrm{C}_{6}$ 碳氢化合物的分 离机理和研究进展, 为开发新型具有良好分离性能的 MOF 材料提供研究思路.
\end{abstract}

关键词 $\mathrm{C}_{4} \sim \mathrm{C}_{6}$ 碳氢化合物; 金属有机框架材料; 吸附; 分离; 纯化

\section{Separation and Purification of $\mathrm{C}_{4} \sim \mathrm{C}_{6}$ Hydrocarbons Using Metal-organic Frameworks}

\author{
Guo, Zhenbin ${ }^{a} \quad$ Zhang, Yuanyuan ${ }^{*}, b \quad$ Feng, Xiao $^{a}$ \\ ( ${ }^{a}$ School of Chemistry and Chemical Engineering, Beijing Institute of Technology, Beijing 100081) \\ ( ${ }^{b}$ Advanced Research Institute of Multidisciplinary Science, Beijing Institute of Technology, Beijing 100081)
}

\begin{abstract}
As important chemical raw materials and energy source, $\mathrm{C}_{4} \sim \mathrm{C}_{6}$ hydrocarbons are mainly used to produce polymer rubber, plastics and high-quality gasoline, which requires high purity of the raw materials. For example, the purity requirement in 1,3-butadiene polymerization reactor is higher than $99.5 \%$. When producing butyl rubber, tert-butylamine, pivalic acid, etc., the purity of isobutylene should surpass $99 \%$. In the traditional petrochemical industry, $\mathrm{C}_{4} \sim \mathrm{C}_{6}$ hydrocarbons are mostly separated and purified through distillation, yet suffering from large energy consumption, high equipment cost and poor economic benefits. Adsorption separation with solid adsorbents can not only reduce energy cost and environmental footprints, but also improve separation efficiency. Metal-organic frameworks (MOFs) are a class of crystalline porous materials assembled from metal ions or clusters and organic linkers. Compared with zeolite, activated carbon and silica gel, MOFs feature high porosity, well-defined open channels, rich functional groups and diverse structures, showing great potentials in gas storage and separation, sensing, catalysis, photoelectric devices, drug release and delivery. Up to now, there have been many reports on separation and purification of $\mathrm{C}_{4} \sim \mathrm{C}_{6}$ hydrocarbons using MOFs by different mechanisms. Specifically, highly selective separation can be achieved by precisely adjusting the size and shape of the MOF channels to match the size of the target molecule. Besides, selecting MOFs with specific functional groups, open metal sites or flexible skeletons to regulate the interactions between the gas molecules and backbone, can also achieve efficient separation. This review introduced the importance of $\mathrm{C}_{4} \sim \mathrm{C}_{6}$ hydrocarbons separation and summarized the current research progress of using MOFs to separate and purify $\mathrm{C}_{4} \sim \mathrm{C}_{6}$ hydrocarbons. In addition, we also summed up the challenges of using MOFs as industrial adsorbents and pointed out the possible research directions in the future, which may provide ideas for designing new MOFs with high performance for crucial separation processes.
\end{abstract}

Keywords metal-organic frameworks; $\mathrm{C}_{4} \sim \mathrm{C}_{6}$ hydrocarbons; adsorption; separation; purification

\section{1 引言}

$\mathrm{C}_{4} \sim \mathrm{C}_{6}$ 碳氢化合物作为石化工业和汽油加工中主 要的能源和原料, 具有重要的商业价值 ${ }^{[1]} . \mathrm{C}_{4}$ 碳氢化合 物主要包括 1,3-丁二烯、正丁烯、异丁烯、正丁烷和异 丁烷. 其中 1,3-丁二烯应用最广、商业价值最大, 主要 用作单体合成顺丁橡胶(CBR, 聚丁二烯)、丁苯橡胶
(SBR, 苯乙烯-丁二烯)、树脂(ABS, 丙烯腈-丁二烯-苯 乙烯)、丁腈橡胶(NBR, 丙烯腈-丁二烯)等, 可应用于轮 胎、粘合剂、尼龙-66 等的制备. 异丁烯和正丁烯是从 石油中提炼出来的重要的石化产品 ${ }^{[2]}$. 每年有千万吨的 异丁烯产出, 最终转化为燃料和塑料; 正丁烯则用来合 成烷基化汽油、清洁剂醇、合成润滑剂和增塑剂等 ${ }^{[1]}$. 汽 油组成中含有 $10 \%$ 的戊烷和已烷，其中双取代的脂肪烃

\footnotetext{
*E-mail: 6120190112@bit.edu.cn

Received March 21, 2020; published April 20, 2020.

Project supported by the National Natural Science Foundation of China (Nos. 21922502, 21674012).

项目受国家自然科学基金(21922502,21674012)资助.
} 
比单取代和直链的烷烃具有更高的辛烷值(RON), 辛烷 值越高, 汽油抗爆性能越好 ${ }^{[3]}$. 例如 2,3-二甲基丁烷和 2,2-二甲基丁烷的辛烷值分别是 105 和 94, 而单取代的 2-甲基戊烷和 3-甲基戊烷辛烷值只有 74 和 75, 直链的 正己烷辛烷值更是低至 30 . 为了满足工业化和商品化 的应用需求, $\mathrm{C}_{4} \sim \mathrm{C}_{6}$ 碳氢化合物的分离具有重要的意 义.

传统工业生产中, $\mathrm{C}_{4}$ 碳氢化合物主要通过流化催化 裂化技术(FCC)、蒸气裂解(Steam cracking)、甲醇一烯烃 过程(MTO)三种工艺产出 ${ }^{[4]}$. 如表 1 所示, 不同的工艺 最后生成 $\mathrm{C}_{4}$ 碳氢化合物的比例有所不同. 最终化学品 的生产, 对原料纯度有严格要求, 例如 1,3-丁二烯聚合 反应器中纯度要求大于 $99.5 \%$; 生产丁基橡胶、叔丁胺、 特戊酸等产品时, 异丁烯的纯度需要大于 $99 \%$. 但由于 $\mathrm{C}_{4}$ 碳氢化合物之间具有相似的物理性质(表 2 ), 分离纯 化难度较大. 工业上, 1,3-丁二烯的分离纯化通常需要 在 $300 \mathrm{kPa}, 323 \sim 393 \mathrm{~K}$ 的条件下对轻烃混合物进行萃 取精馏, 此过程需要高塔板数的精馏塔(超过 110 个塔 板)和乙腈、 $N, N$-二甲基甲酰胺等大量有机溶剂 ${ }^{[5]}$. 异丁 烯多是通过在弱酸条件下选择性的形成甲基叔丁基醚 或叔丁醇进行分离 ${ }^{[6]}$. $\mathrm{C}_{6}$ 脂肪烃类碳氢化合物, 有五种 同分异构体(正己烷、2-甲基戊烷、3-甲基戊烷、2,2-二 甲基丁烷和 2,3-二甲基丁烷), 通过催化异构化反应大规 模生成. 在该热力学控制的产品流中, 这 5 种同分异构 体的占比都在 $10 \% \sim 30 \%{ }^{[3]}$. 为了获得高辛烷值的混合 物, 目前的工艺普遍是用沸石耖分出正己烷, 得到其它 4 种异构体的混合物(RON 接近 83), 然后将正己烷重新 填入到异构化反应器中继续反应. 此外, 一些分离过程 还通过从双取代甲基丁烷产品中蒸馏分离出单取代甲 基丁烷, 以得到更高质量级别的混合物. 这些分离过程 不仅能耗高, 所需设备成本高, 并且排放大量二氧化碳, 对环境不友好.

表 1 不同工艺产出 $\mathrm{C}_{4}$ 气体的摩尔百分比

Table 1 Composition of $\mathrm{C}_{4}$ stream obtained from different processes

\begin{tabular}{cccc}
\hline Compound & FCC & MTO & Steam cracking \\
\hline i-Butane & $35 \sim 45$ & 0.2 & $0 \sim 2$ \\
n-Butane & $7 \sim 42$ & 4 & $2 \sim 5$ \\
i-Butene & $10 \sim 20$ & $2 \sim 4$ & $18 \sim 32$ \\
1-Butene & $9 \sim 12$ & $20 \sim 26$ & $14 \sim 22$ \\
2-Butene & $20 \sim 29.5$ & $65 \sim 70$ & $5 \sim 15$ \\
Butadiene & $0 \sim 0.5$ & - & $35 \sim 50$ \\
\hline
\end{tabular}

与目前大规模工业蒸馏过程相比，吸附式分离中多 孔材料可以利用客体分子的物理和化学性质不同(如分 子动力学直径、极化率、酸碱性、偶极矩、四极矩、配 位能力等), 实现选择性分离, 可通过与现有分离技术 进行联用甚至替代，从而大幅减少能耗和分离成本. 金 属有机框架 ${ }^{[7]}$ (metal-organic frameworks, MOFs)是一类 由金属离子或团簇和有机单体组装而成的晶态多孔材 料，具有高孔隙率、规整开放的孔道、丰富的官能团和 多样的结构, 在气体储存与分离 ${ }^{[8]}$ 、传感 ${ }^{[9]}$ 、催化 ${ }^{[10]}$ 、 药物释放与传递 ${ }^{[11]}$ 等方向有着巨大的应用前景. 与沸石 分子篮和活性炭相比, MOFs 具有规整开放的孔道, 且 孔道尺寸和化学环境易调控, 部分骨架结构有一定柔 性, 在分离纯化轻烃分子上更具优势. MOFs 用于气体 分离主要通过三种机制: 尺寸篮分、热力学平衡分离和 动力学驱动分离 ${ }^{[2,12]}$. 孔径通常是影响小分子分离的主 要参数, 尺寸笁分主要根据 MOF 材料的孔径大小和气 体分子间动力学直径的差异进行分离. 气体分子的动力 学直径若大于 MOF 材料的孔径尺寸, 则被阻挡在孔道 之外，而尺寸小的分子可以顺利通过. 这种分离机制主 要发生在沸石分子篮和具有刚性结构的 MOF 材料上. 如果 MOF 材料的孔径尺寸较大, 足以容纳所有气体分 子, 则气体的竞争性吸附受热力学平衡效应的影响. 热 力学平衡效应是指当气体分子对孔道内壁的相互作用 较强时, 会优先吸附在孔道内壁上, 这种相互作用主要 取决于吸附剂的孔道表面和吸附质的性质, 如极化率、 磁化率、偶极矩、四极矩、酸碱性等. 动力学控制的分 离指的是当吸附剂对混合物的不同组分的吸附量大致 相同时, 对于某些组分的吸附速率高于其他组分, 由此 实现不同组分的分离纯化. 除了这三种主要的分离机制 外, MOF 材料还有一些特殊的分离机制, 例如当吸附剂 的骨架具有柔性时，在一定温度下，吸附质被累积吸附 到特殊压力点后, 会出现 “gate-opening” 效应, 此时 MOF 骨架孔道被撑开, 吸附量急剧上升. 当孔道的开口 大小在外界刺激下(如温度、压力、客体分子等)可以发 生可逆的变化, 称之为 “呼吸效应” (图 1) ${ }^{[2,12]}$. 无论是 “gate-opening” 效应还是 “呼吸效应”, 都是由于 MOF 材料在外界条件刺激下, 骨架孔道的尺寸和结构发生变 化，导致前后吸附的差异. 除此之外，某些 MOF 材料拥 有离散空腔的孔道, 可以诱导孔道内客体分子的构象发 生变化, 产生特异性吸附 ${ }^{[24]}$.

吸附过程中气体分子受到和多孔材料内表面的相 互作用, 运动速度降低, 释放出热量, 故吸附是放热过

表 $2 \mathrm{C}_{4}$ 碳氢化合物的物理性质对比

Table 2 Physical properties of $\mathrm{C}_{4}$ hydrocarbons

\begin{tabular}{ccccc}
\hline & 沸点 $/{ }^{\circ} \mathrm{C}$ & 偶极矩/D & 极化率 $/ \AA^{3}$ \\
\hline i-Butane & -11.5 & 0.132 & 7.595 & 四极矩/D $\AA$. \\
n-Butane & -0.5 & 0 & 0.638 & 0.433 \\
i-Butene & -6.6 & 0.55 & 7.347 & 7.351 \\
1-Butene & -6.5 & 0.359 & -2.006 & 7.624 \\
Butadiene & -4.7 & 0 & -1.876 & -5.469 \\
\hline
\end{tabular}




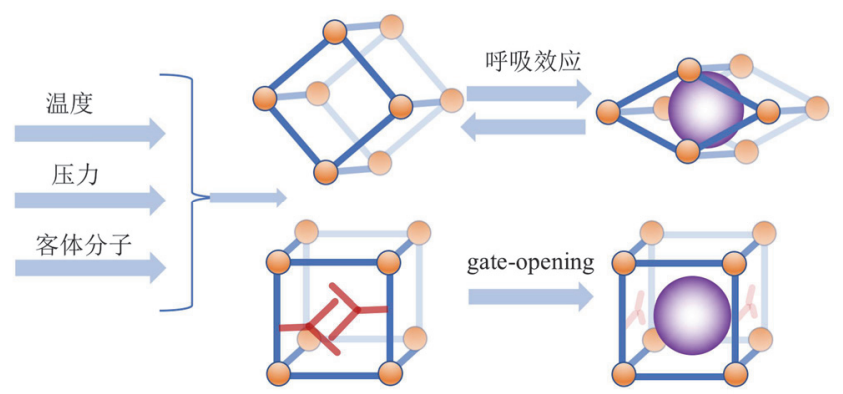

图 1 “呼吸效应” 和 “gate-opening” 效应示意图.

Figure 1 Schematic diagram of "breathing" and "gate-opening" effect.

程; 反之, 脱附是吸热过程. 在吸脱附过程中, 往往伴 随着温度的变化. 不同温度下, 多孔材料对吸附质的吸 附量大小有所不同，根据不同温度下的吸附等温线，可 以计算得到吸附焓. Eq. 1 为 Clausius-Clapeyron 等量吸 附热的计算方程, 其中 $q_{\mathrm{st}}$ 为等量吸附热, $R$ 为气体常数, $T$ 代表温度, $P$ 代表压力, $n$ 代表气体吸附量 ${ }^{[13]}$. 吸附焓 是气体分离过程中关键的热力学变量, 决定了气体吸脱 附过程中吸附剂内部温度变化的程度. 温度变化涉及气 体分子动力学和吸附剂吸附平衡, 影响着最终的分离性 能.

$$
q_{s t}=R T^{2}\left(\frac{\partial \ln P}{\partial T}\right)_{n}
$$

本综述将主要介绍目前 $\mathrm{MOF}$ 材料在 $\mathrm{C}_{4} \sim \mathrm{C}_{6}$ 碳氢化
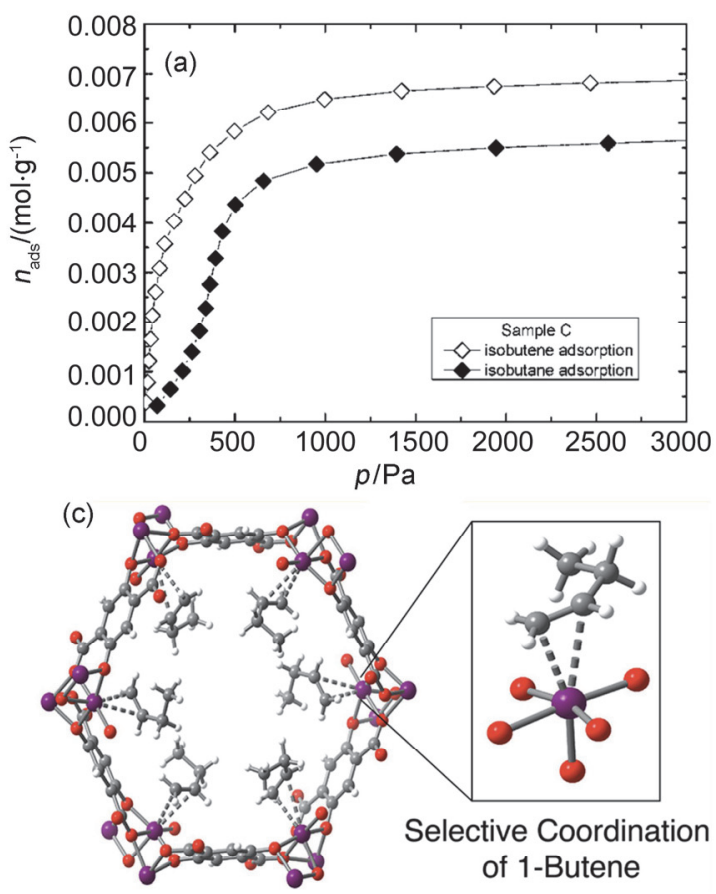

合物分离纯化中的研究进展，探讨 MOF 材料的孔道尺 寸和形状、骨架结构、官能团、开放金属位点等对分离 过程的影响. 与此同时, 还总结了目前 MOF 材料作为 吸附剂存在的不足之处以及未来的研究方向, 为进一步 开发新型的具有优异分离性能的 MOF 材料提供研究思 路.

\section{$2 \mathrm{C}_{4}$ 碳氢化合物的分离}

$\mathrm{C}_{4}$ 碳氢化合物中, 烯烃具有较高的商业价值, $\mathrm{C}_{4}$ 气 体的分离, 主要是将 $\mathrm{C}_{4}$ 烯烃从不同的 $\mathrm{C}_{4}$ 气体混合物中 单独提取出来. 相对于 $\mathrm{C}_{2} 、 \mathrm{C}_{3}$ 碳氢化合物, $\mathrm{C}_{4}$ 烯烃除了 具有相似的物理性质, 还存在顺反异构, 增加了其分离 的难度.

通过利用 MOFs 中的开放金属位点与不饱和烯烃产 生 $\pi$ 络合相互作用，可以实现选择性的 $\mathrm{C}_{4}$ 吸附 ${ }^{[14 \sim 18]}$. 2008 年, Hartmann 等 ${ }^{[14]}$ 使用具有开放金属位点的 HKUST-1 对异丁烯和异丁烷进行吸附测试, 发现 HKUST-1 对于异丁烯的吸附量明显较高(图 2a). 穿透实 验表明(图 2b), 在低压下有利于两者的分离, 在物质的 量比为 $1: 1$ 时其分离系数为 2.1 . 最近, Long 等 ${ }^{[16]}$ 报道 了两种具有高密度开放金属位点的 MOF 材料一一 $\mathrm{Co}_{2}$ (m-dobdc)和 $\mathrm{Ni}_{2}(\mathrm{~m}-\mathrm{dobdc})\left(\mathrm{m}-\mathrm{dobdc}^{4-}\right.$ : 4,6-二羟基间 苯二甲酸), 通过同步 $\mathrm{X}$ 射线衍射数据分析晶体结构和 气体分子在孔道中的状态, 发现与 2-丁烯相比, 正丁烯

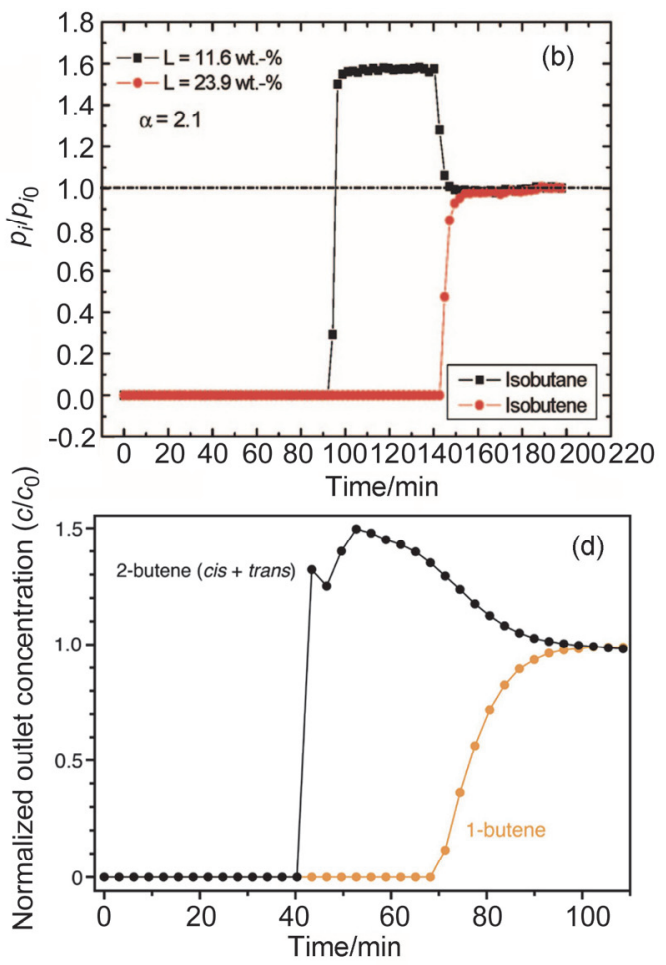

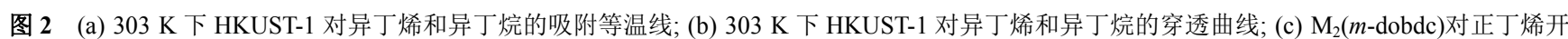
放金属位点示意图; (d) $328 \mathrm{~K} \mathrm{下} \mathrm{Ni}_{2}$ (m-dobdc)对正丁烯和 2-丁烯的穿透曲线. (改编自文献[14]、[16])

Figure 2 (a) Comparison of isobutene and isobutane adsorption isotherms at $303 \mathrm{~K}$ on HKUST-1. (b) Breakthrough curves for the separation of an isobutene/isobutane mixture over HKUST-1 at $303 \mathrm{~K}$. (c) Views of the active metal sites of $\mathrm{M}_{2}$ (m-dobdc) on 1-butene. (d) Breakthrough curves of $\mathrm{Ni}_{2}(\mathrm{~m}$-dobdc) to an equimolar feed of 1-butene and 2-butene at $328 \mathrm{~K}$. (Adapted from the literature [14], [16]) 
的乙基能够更加自由延伸到框架的一维通道中, 其双键 与金属开放位点距离更近, 相互作用更强(图 2c). 穿透 实验结果表明, $\mathrm{Ni}_{2}$ (m-dobdc)能够在 $328 \mathrm{~K}$ 下成功将等物 质的量比的 2-丁烯和正丁烯进行分离(图 2d).

根据孔径大小和气体动力学直径进行尺寸篮分, 是 最直接有效的分离方式. 2016 年, 何亚兵课题组 ${ }^{[19]}$ 通过 烷氧基链将两个 $\left[1,1^{\prime}: 4^{\prime}, 1^{\prime \prime}\right.$-三联苯]-3,3",5,5"-四羧酸配体 的苯环进行交联, 利用交联后的八羧酸配体, 制备了 MOF-ZJNU-80. 与未交联的 MOF 相比, MOF-ZJNU-80 在保持拓扑结构的同时成功实现了孔道分割, 孔径尺寸 变小, 改变了正丁烯分子在孔道中的填充效率, 大大提 升了正丁烯分子的吸附量. 2017 年, 邢华斌课题组 ${ }^{[20]}$ 合 成了三种阴离子柱穿插结构的超微孔 MOF 材料 GeFSIX-2-Cu-i、NbFSIX-2-Cu-i、GeFSIX-14-Cu-i(图 3a〜 $3 \mathrm{~d})$. 通过改变配体和置换阴离子柱, MOFs 的空腔尺寸 可以在 $0.02 \mathrm{~nm}$ 的范围内进行精准的调控, 从而对不同 $\mathrm{C}_{4}$ 烯烃进行尺寸篎分. 此外, 这些结构中的有机配体可 以发生一定角度的旋转, 使得 MOFs 形成一种收缩的柔 性孔窗, 从而进行分子识别. 结合分子识别和尺寸篮分 效应, 邢华斌团队对多组分混合物 $\left(\mathrm{C}_{4} \mathrm{H}_{6} / n-\mathrm{C}_{4} \mathrm{H}_{8} /\right.$ iso$\mathrm{C}_{4} \mathrm{H}_{8} / \mathrm{He}, 50 / 15 / 30 / 5$, 物质的量比) 和双组分混合物 $\left(n-\mathrm{C}_{4} \mathrm{H}_{8} /\right.$ iso $-\mathrm{C}_{4} \mathrm{H}_{8}, 50 / 50$, 物质的量比)进行了穿透实验. 首先, 根据样品密度和粒径的不同, 将一定量的 MOF 材料分别填充到长度为 $50 \mathrm{~mm}$ 、内径为 $5.5 \mathrm{~mm}$ 的不锈
钢管路中，然后将混合气以 $0.5 \mathrm{~mL} / \mathrm{min}$ 的流速通过，后 端用气相色谱进行检测. 结果发现, 这三种 MOFs 均能 够成功将混合气中的各组分分离(图 $3 \mathrm{e} \sim 3 \mathrm{~g}$ ). 对材料加 热活化后进行循环吸附测试，发现在 6 个循环后，材料 依旧能够保持良好的吸附分离性能. 2019 年，邢华斌课 题组 ${ }^{[21]}$ 又开发了一种柔性 MOF- - MnINA, 它拥有周 期性口袋状的空腔, 并由狭窄的瓶颈相连形成一维贯穿 孔道 (图 4a). 由于 $\mathrm{Mn}^{2+}$ 与 $\mathrm{INA}^{-}$之间的键长较短, MnINA 的孔径尺寸只有 $0.462 \mathrm{~nm}$, 处于正丁烯和异丁 烯的分子动力学直径之间 $\left(n-\mathrm{C}_{4} \mathrm{H}_{8}: 0.446 \mathrm{~nm}\right.$, iso $-\mathrm{C}_{4} \mathrm{H}_{8}$ : $0.484 \mathrm{~nm})$. 根据尺寸篮分效应，正丁烯很容易插入到孔 道中，而异丁烯被排除在外。根据理想吸附溶液理论 (IAST)计算, 低压下, MnINA 对正丁烯/异丁烯的选择性 高达 327.7. 需要指出的是, 由于有机连接单体一定角 度的旋转, 使得 MnINA 吸附异丁烯到 $10 \mathrm{kPa}$ 时, 出现 “gate-opening” 效应, 异丁烯吸附量急剧上升(图 4b). 穿 透实验表明, MnINA 能够有效地分离 $n-\mathrm{C}_{4} \mathrm{H}_{8}$ 和 iso $-\mathrm{C}_{4} \mathrm{H}_{8}$, 并保持良好的循环性能(图 4c, 4d).

借助柔性 MOF 材料在客体分子作用下发生孔道结 构变化的特性, 也可实现 $\mathrm{C}_{4}$ 气体的分离. 2014 年, Glaser 等 ${ }^{[22]}$ 利用柔性 $\mathrm{Cu}-\mathrm{MOF}$ 在 $300 \mathrm{kPa}$ 的高压和不同温度下 对正丁烷、正丁烯、异丁烷和异丁烯进行单组分吸附测 试，发现只有正丁烯能够使柔性孔完全撑开，产生 “gate-opening” 效应, 而其它三种不能.
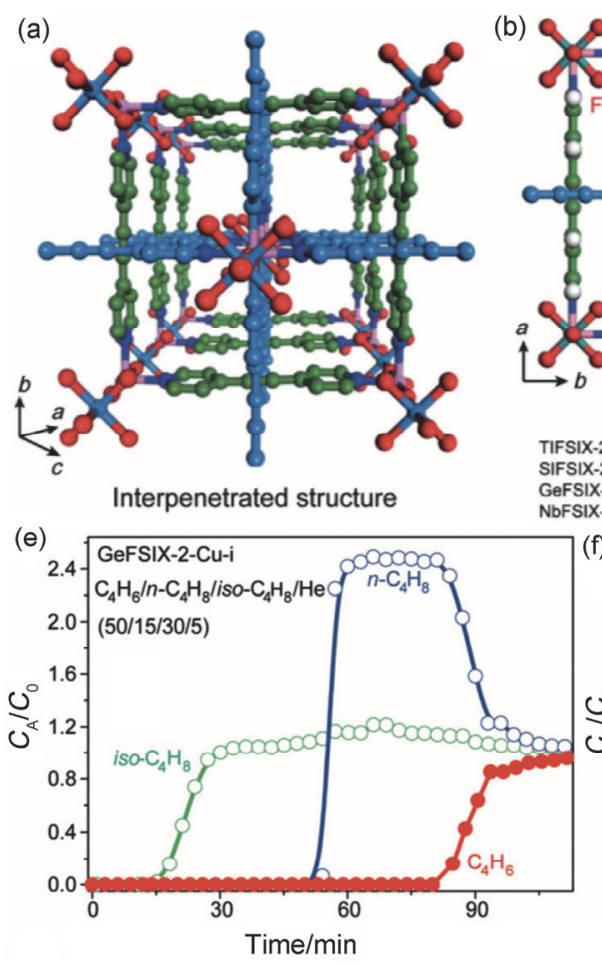

(b)

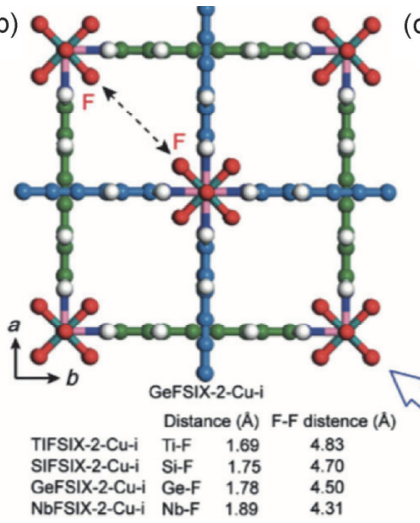

(c)

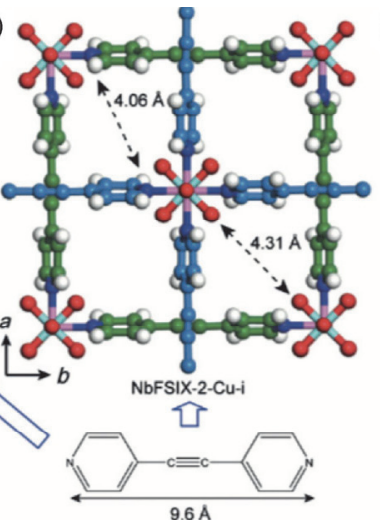

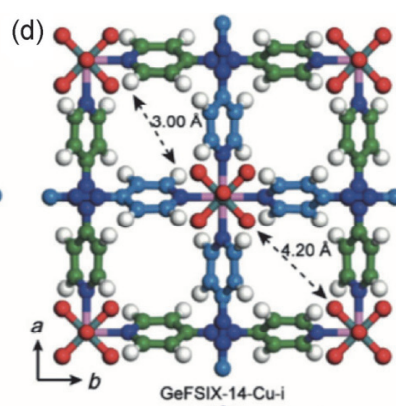

GeFSIX-14-Cu-i

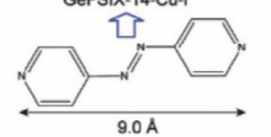

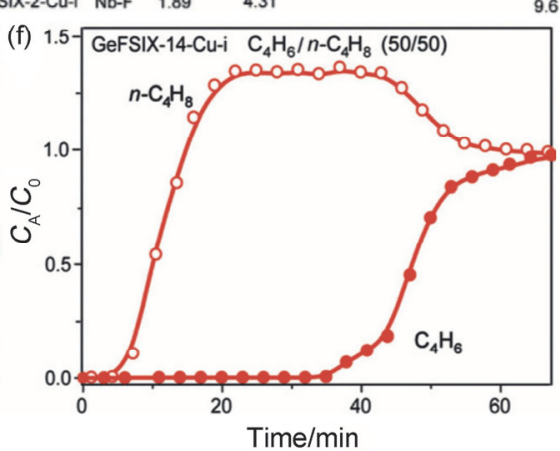

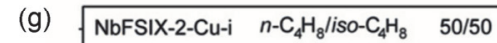

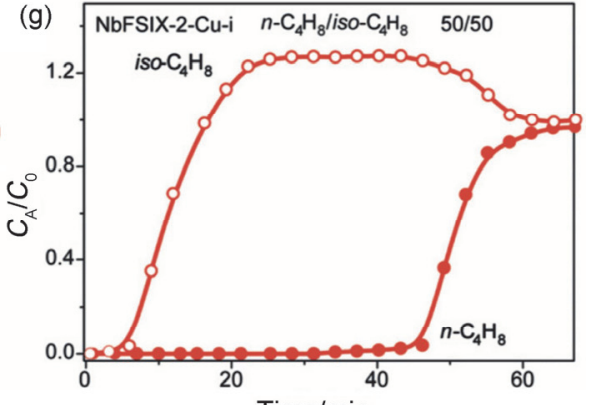

图 3 (a) MFSIX- $n$-Cu-i 结构示意图; (b) GeFSIX-2-Cu-i; (c) NbFSIX-2-Cu-i; (d) GeFSIX-14-Cu-i; (e) GeFSIX-2-Cu-i 对多组分混合物 $\left(\mathrm{C}_{4} \mathrm{H}_{6} / n-\mathrm{C}_{4} \mathrm{H}_{8} / i s o-\mathrm{C}_{4} \mathrm{H}_{8} / \mathrm{He}, 50 / 15 / 30 / 5\right)$ 的穿透曲线; (f) GeFSIX-14-Cu-i 对双组分混合物 $\left(n-\mathrm{C}_{4} \mathrm{H}_{8} / i s o-\mathrm{C}_{4} \mathrm{H}_{8}, 50 / 50\right)$ 的穿透曲线; ( $\mathrm{g}$ ) NbFSIX-2-Cu-i 对双 组分混合物 $\left(n-\mathrm{C}_{4} \mathrm{H}_{8} / i s o-\mathrm{C}_{4} \mathrm{H}_{8}, 50 / 50\right)$ 的穿透曲线. (改编自文献[20])

Figure 3 (a) Schematic representations of the interpenetrated structure; (b) GeFSIX-2-Cu-i; (c) NbFSIX-2-Cu-i and (d) GeFSIX-14-Cu-i; (e) breakthrough curves for the mixture $\left(\mathrm{C}_{4} \mathrm{H}_{6} / n-\mathrm{C}_{4} \mathrm{H}_{8} / i\right.$ so- $\left.\mathrm{C}_{4} \mathrm{H}_{8} / \mathrm{He}, 50 / 15 / 30 / 5\right)$ over GeFSIX-2-Cu-i; (f) breakthrough curves for $n$ - $\mathrm{C}_{4} \mathrm{H}_{8} / i$ so- $\mathrm{C}_{4} \mathrm{H}_{8}(50 / 50)$ over GeFSIX-14-Cu-i; (g) breakthrough curves for $n-\mathrm{C}_{4} \mathrm{H}_{8} /$ iso- $\mathrm{C}_{4} \mathrm{H}_{8}(50 / 50)$ over NbFSIX-2-Cu-i. (Adapted from the literature [20]) 
(a)

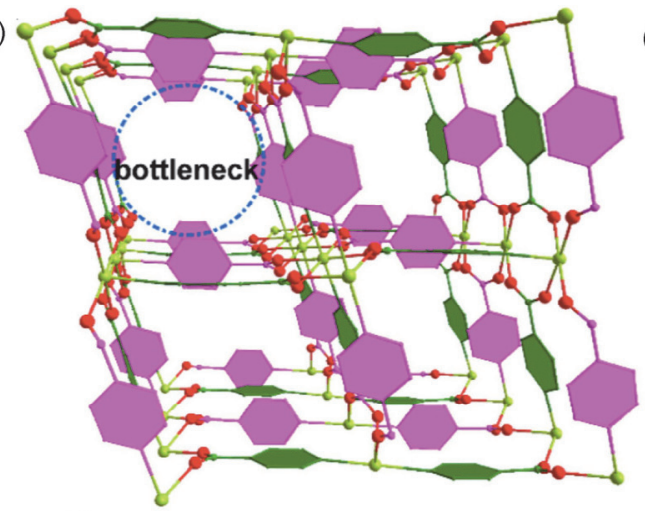

(c)

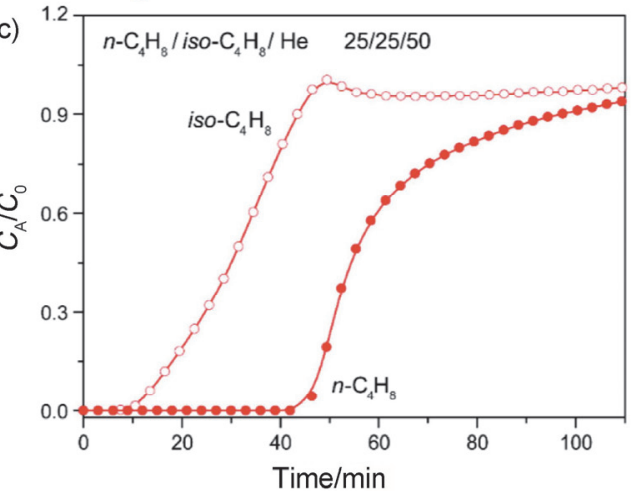

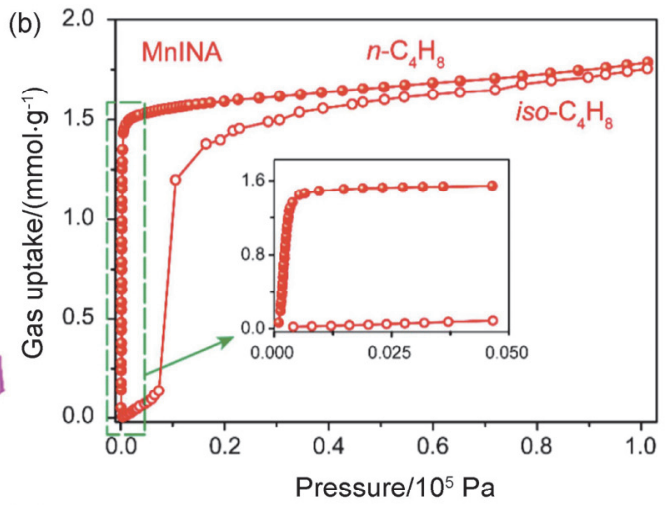

(d)

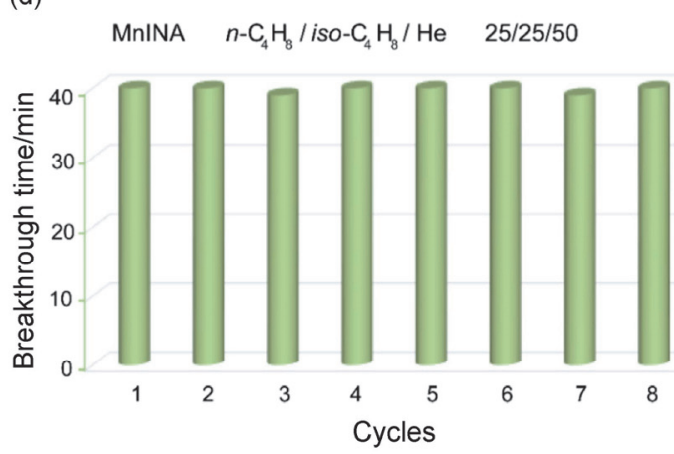

图 4 (a) MnINA 的孔道示意图; (b) MnINA 对正丁烯和异丁烯的吸附等温线; (c) MnINA 对正丁烯和异丁烯的穿透曲线; (d) MnINA 对正丁烯和异 丁烯穿透循环时间柱状图. (改编自文献[21])

Figure 4 (a) Perspective view of the channels of MnINA; (b) the $n-\mathrm{C}_{4} \mathrm{H}_{8}$ and $i s o-\mathrm{C}_{4} \mathrm{H}_{8}$ adsorption isotherms on MnINA at $298 \mathrm{~K}$; (c) experimental breakthrough tests for $n$ - $\mathrm{C}_{4} \mathrm{H}_{8} /$ iso- $\mathrm{C}_{4} \mathrm{H}_{8} / \mathrm{He}(25 / 25 / 50)$ separation on MnINA; (d) cyclic column breakthrough tests for $n-\mathrm{C}_{4} \mathrm{H}_{8} / i s o-\mathrm{C}_{4} \mathrm{H}_{8} / \mathrm{He}(25 / 25 / 50)$ on MnINA. (Adapted from the literature [21])

(a)
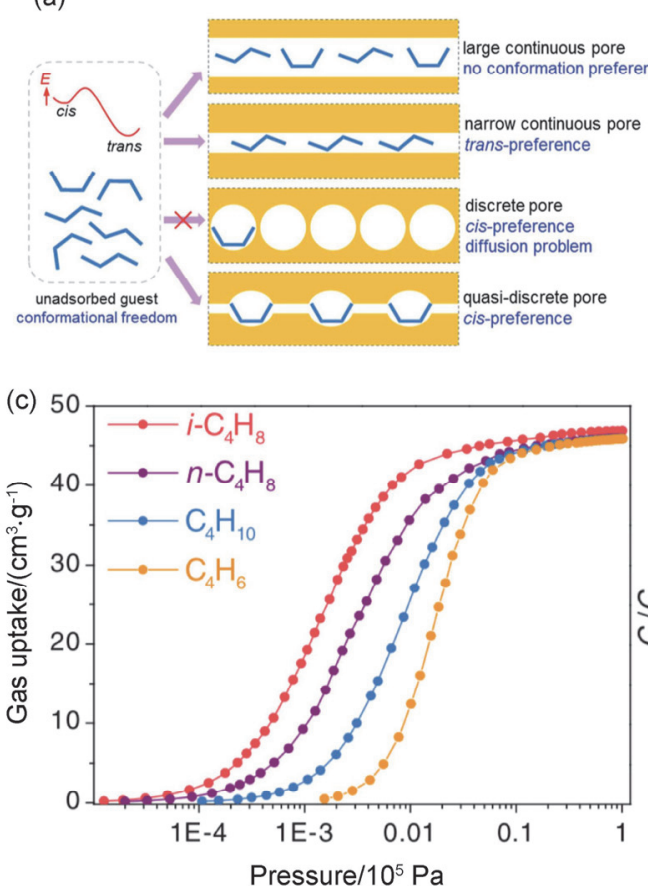
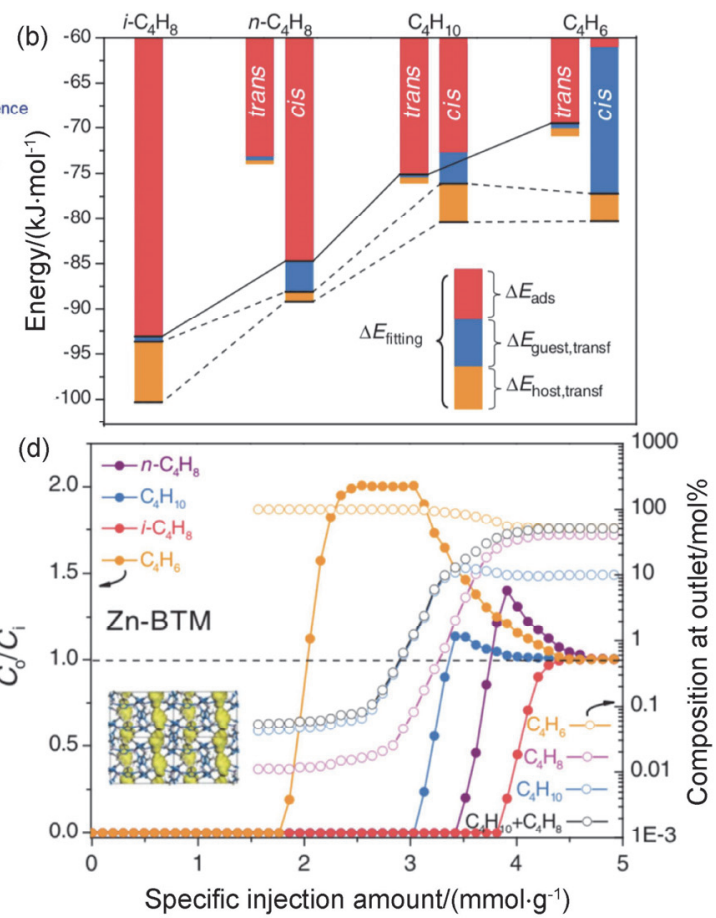

图 5 (a)不同类型孔道吸附 $\mathrm{C}_{4}$ 碳氢化合物的示意图; (b)密度泛函理论计算 Zn-BTM 对 $\mathrm{C}_{4}$ 碳氢化合物的吸附焓; (c) $298 \mathrm{~K}$ 下 Zn-BTM 对 $\mathrm{C}_{4}$ 碳氢化 合物的吸附等温线; (d) Zn-BTM 对 $\mathrm{C}_{4}$ 碳氢化合物 $\left(\mathrm{C}_{4} \mathrm{H}_{6} / n-\mathrm{C}_{4} \mathrm{H}_{8} / i s o-\mathrm{C}_{4} \mathrm{H}_{8} / \mathrm{C}_{4} \mathrm{H}_{10}, 5 / 2 / 2 / 1\right)$ 的穿透曲线. (改编自文献[24])

Figure 5 (a) Schematic diagram of adsorption of $\mathrm{C}_{4}$ hydrocarbons by different types of channels; (b) PDFT-derived thermodynamic energies for adsorption of $\mathrm{C}_{4}$ hydrocarbons in $\mathrm{Zn}$-BTM; (c) Adsorption isotherms for $\mathrm{C}_{4} \mathrm{H}_{6}, n-\mathrm{C}_{4} \mathrm{H}_{8}, i-\mathrm{C}_{4} \mathrm{H}_{8}$, and $\mathrm{C}_{4} \mathrm{H}_{10}$ in Zn-BTM at $298 \mathrm{~K}$; (d) Breakthrough curves for $\mathrm{C}_{4} \mathrm{H}_{6} / n-\mathrm{C}_{4} \mathrm{H}_{8} /$ iso- $\mathrm{C}_{4} \mathrm{H}_{8} / \mathrm{C}_{4} \mathrm{H}_{10}(5 / 2 / 2 / 1)$ over $\mathrm{Zn}-\mathrm{BTM}$. (Adapted from the literature [24]) 
2016 年, Kitagawa ${ }^{[23]}$ 报道了一种三重穿插结构的柔 性 MOF-SD-65. SD-65 拥有离散的空腔, 吸附不同 $\mathrm{C}_{4}$ 气 体后进行原位 X 射线粉末衍射和固体核磁的表征, 发现 1,3-丁二烯能选择性地吸附在孔腔内, 并诱导骨架发生 “gate-opening” 效应. 在 $25{ }^{\circ} \mathrm{C}$ 下进行穿透实验, 将 95 $\mathrm{wt} \%$ 的 SD-65 和 $5 \mathrm{wt} \%$ 的石墨混合压片，研磨造粒，笁 选出粒径在 $0.4 \sim 2.2 \mathrm{~mm}$ 范围内的复合材料填充在长度 为 $200 \mathrm{~mm}$ 、内径为 $0.5 \mathrm{inch}$ 的不锈钢管路中, 混合气体 以 $10 \mathrm{~mL} / \mathrm{min}$ 的流速通过样品, 后端的流出气用气相色 谱每 $3 \mathrm{~min}$ 进行一次检测. 结果显示, SD-65 能够成功从 含有反-2-丁烯、顺-2-丁烯、正丁烷、正丁烯、异丁烷、 异丁烯和 1,3-丁二烯七种组分的混合物中 $(40 \%$ 二二烯、 $49 \%$ 丁烯混合物、11\%丁烷混合物)分离出丁二烯.

2017 年, 张杰鹏课题组 ${ }^{[24]}$ 报道了一种具有连续离 散空腔孔道的 MOF 材料-Zn-BTM, 这种离散空腔的孔 道可以诱导 1,3-丁二烯发生构象变化, 使其在孔道内由 反式构象转变为顺式构象, 即稳态构象转变为亚稳态构 象. 利用客体分子结构变化的能量差异来降低 1,3-丁二 烯和 MOF 孔道内壁的相互作用(图 $5 \mathrm{a} \sim 5 \mathrm{c}$ ). 在这项工 作中, 张杰鹏团队选择了 10 种 MOF 材料(4 种具有开放 金属位点、 4 种亲水性 MOFs、2 种疏水性 MOFs), 并在 室温下对物质的量比为 $5: 2: 2: 1$ 的 $\mathrm{C}_{4}$ 混合物 $\left(\mathrm{C}_{4} \mathrm{H}_{6} / n-\mathrm{C}_{4} \mathrm{H}_{8} /\right.$ iso- $\left.\mathrm{C}_{4} \mathrm{H}_{8} / \mathrm{C}_{4} \mathrm{H}_{10}\right)$ 进行动态穿透实验. 在穿 透实验之前, 张杰鹏团队首先将装有 $\mathrm{MOF}$ 材料的不锈 钢管路 (长度为 $10 \mathrm{~cm}$ 、内径为 $0.46 \mathrm{~cm}$ ) 在 $423 \mathrm{~K}$ 下通入 氦气 $10 \mathrm{~h}$, 然后控制混合气以 $2 \mathrm{~mL} / \mathrm{min}$ 的速度流经样 品. 结果显示, $\mathrm{C}_{4} \mathrm{H}_{6}$ 能够在 $\mathrm{Zn}-\mathrm{BTM}$ 中优先洗脱出来, 其后依次是 $\mathrm{C}_{4} \mathrm{H}_{10} 、 n-\mathrm{C}_{4} \mathrm{H}_{8} 、$ iso- $\mathrm{C}_{4} \mathrm{H}_{8}$, 最终 1,3-丁二烯 的纯度能够达到 99.5\%(图 5d). 这种优先洗脱 1,3-丁二 烯的方法, 避免了吸附后活化时丁二烯加热自聚, 高效 实现了 1,3-丁二烯的分离纯化.

\section{$3 \mathrm{C}_{6}$ 碳氢化合物的分离}

$\mathrm{C}_{5} \sim \mathrm{C}_{7}$ 的双支链烷烃是高质量的汽油主要成分. 一般烷烃支链化程度越大, 其辛烷值越高, 汽油的防爆 防震能力越好. 因此, 烷烃的直链、单取代、双取代异 构体的分离在石油工业中十分重要.

为了制备多取代烷烃, 工业上是将直链烷烃进行催 化异构化反应, 但由于热力学平衡的限制, 异构化的产 物通常由未反应的直链烷烃及单支链异构体和双支链 异构体组成. 在当前的工业生产中, 未反应的直链烷烃 一般通过 $5 \AA$ 分子篮进行分离, 然后再次导入到催化异 构化反应器中, 由此循环最终得到单取代和多取代烷烃 的混合物. 这一过程存在的问题是 $5 \AA$ 分子笁并不能分 离单支链异构体和多支链异构体, 无法进一步提高汽油 的质量. 由于烷烃异构体化学惰性相同, 极化率、沸点 等相近, 只有在动力学直径上有所差别, 所以 $\mathrm{C}_{6}$ 烷烃的 分离, 大多基于尺寸篮分或者分子识别.
迄今为止, 将 MOFs 应用于异构化烷烃的分离已有 很多研究，并取得了良好的分离效果 ${ }^{[25 ~ 30]}$. 2006 年，陈 邦林课题组 ${ }^{[25]}$ 报道了一种三维 MOF 材料- - MOF-508, 并将其应用于己烷直链烷烃和支链烷烃异构体的分离. MOF-508 具有双重穿插结构, 且骨架中的溶剂分子可 使其晶态结构发生可逆变化(图 6a，6b). MOF-508 中一 维孔道尺寸为 $0.4 \mathrm{~nm} \times 0.4 \mathrm{~nm}$, 根据烷烃异构体动力学 直径的不同以及和孔壁范德华相互作用的差异，戊烷和 己烷的直链烷烃可被吸附在孔道中，而支链异构体则被 排除在外．在穿透实验中，正戊烷、正己烷、2-甲基丁 烷、2,2-二甲基丁烷和 2-甲基戊烷成功得到分离(图 6c).
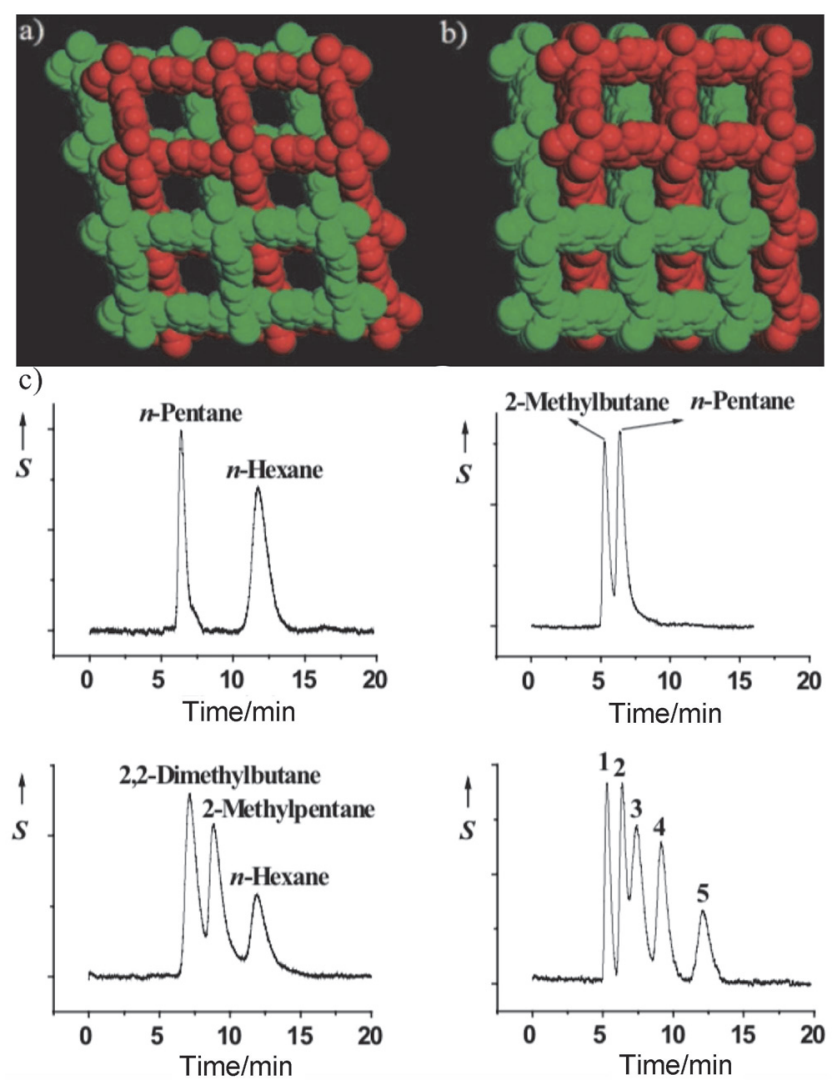

图 6 (a) MOF-508a 空间填充示意图; (b) MOF-508b 空间填充示意图; (c) MOF-508 色谱柱分离烷烃混合物的色谱图(右下: 1: 2-甲基丁烷; 2: 正戊烷；3：2,2-二甲基丁烷；4：2-甲基戊烷；5：正己烷). (改编自文献 [25])

Figure 6 (a) Space-filling representations of the structures of MOF-508a (the open phase); (b) Space-filling representations of the structures of MOF-508b (the dense phase); (c) Chromatograms of alkane mixtures separated on a MOF-508 column [bottom right: 2-methylbutane (1), n-pentane (2), 2,2-dimethylbutane (3), 2-methylpentane (4), and $n$-hexane (5)]. (Adapted from the literature [25])

MOF 材料可以提供沸石分子篮或者其它多孔介质 难以实现的孔道结构特征，可实现不同几何形状分子的 篮分. 2013 年, Long 等 ${ }^{[26]}$ 报道了一种 MOF 材料一一 $\mathrm{Fe}_{2}(\mathrm{BDP})_{3}\left(\mathrm{BDP}^{2-}: 1,4\right.$-苯二吡唑羧酸), 其金属节点具有 较高的配位数, 与有机配体组装形成三角形状的一维孔 道结构(图 7a). $\mathrm{Fe}_{2}(\mathrm{BDP})_{3}$ 三角形的孔道可以容纳所有己 烷异构体, 由于直链的正己烷比支链己烷异构体具有更 
高的柔性, 大部分的表面可与 MOF 孔道内壁接触产生 范德华相互作用, 更易吸附在孔道内(图 7b). Long 等分 别在 130、160 和 $200{ }^{\circ} \mathrm{C}$ 下进行了己烷 5 种同分异构体 的单组分吸附测试, 结果表明这种独特的孔结构能够最 大限度地提高孔壁与正己烷的范德华相互作用, 达到较 高吸附量, 随着支链化程度的增加, 范德华相互作用越 来越小, 其吸附量越来越低, 由此实现分离(图 7c). 在 $160{ }^{\circ} \mathrm{C}$, 对 5 种等物质的量己烷异构体的混合气体进行 穿透实验, 首先将定量的 $\mathrm{Fe}_{2}(\mathrm{BDP})_{3}$ 填入到内径为 0.95 $\mathrm{cm}$ 的 $\mathrm{U}$ 形玻璃管中, 然后以干燥 $\mathrm{N}_{2}$ 作为载气将一定比 例的已烷异构体的混合相以 $2.5 \mathrm{~mL} / \mathrm{min}$ 的速度通过 $U$ 形管, 检测后端各组分比例. 结果显示 $\mathrm{Fe}_{2}(\mathrm{BDP})_{3}$ 成功 将双取代异构体、单取代异构体、正己烷分离, 即 2,2二甲基丁烷和 2,3-二甲基丁烷最先流出, 其次是 2-甲基 戊烷和 3-甲基戊烷，最后流出的是正己烷(图 7d).

由对苯二甲酸类和金属配位而成的 MIL-53 和 MIL-88B 系列, 是典型的具有 “呼吸效应” 的 MOF 材 料, 通过在配体上进行官能团修饰, 能显著影响框架的 柔性, 展现出对烷烃不同的分离性质. 2014 年, Silva 等 ${ }^{[27]}$ 报道了在 MIL-53(Fe) 的配体上修饰 $-\mathrm{CF}_{3}$, 使其孔径 从 $0.76 \mathrm{~nm}$ 减小到 $0.42 \sim 0.56 \mathrm{~nm}$. 通过尺寸篎分和骨架 柔性的结合, 成功实现 2,2-二甲基丁烷、正己烷和 3-甲
基戊烷的分离, 对提高 RON 值具有重要意义.

2015 年, Eddaoudi 等 ${ }^{[28]}$ 设计合成了一系列基于稀土 金属的 MOF 材料—— fcu-MOF，其孔窗开口尺寸为 0.47 $\mathrm{nm}$, 稍大于直链烷烃，小于支链烷烃异构体，能有效分 离 $\mathrm{C}_{5}$ 直链和支链烷烃. 2018 年, 李静课题组 ${ }^{[29]}$ 通过选择 具有不同分子尺寸的四羧酸配体合成了三种 $\mathrm{Zr}$ 基 $\mathrm{MOF}$ 材料—Zr-bptc (bptc: 3, 3',5,5'-联苯四甲酸)、Zr-abtc

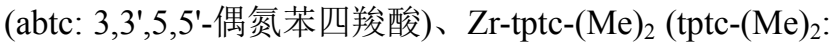
$2^{\prime}, 5^{\prime}$-二甲基-[1, $1^{\prime}: 4^{\prime}, 1^{\prime \prime}$-三联]-3,3",5,5"-四羧酸), 以此调 控相邻 SBU之间的距离和孔径大小. Zr-bptc 含有立方笼 状孔，顶点上是 $\mathrm{Zr}_{6}$ 簇，面上由 $\mathrm{bptc}^{4-}$ 配体连接，其笼状 孔腔尺寸约为 $1.2 \mathrm{~nm}$, 立方结构的十二个 “边窗” 开口 尺寸约为 $0.45 \mathrm{~nm}$ (图 8a). 由于这种特殊的笼状孔结构, 孔腔可容纳大量的吸附质，而小的 “边窗” 开口有效限 制了吸附质的扩散，使得在相同条件下, Zr-bptc 对正己 烷的吸附量是 $5 \AA$ 分子篮的 1.7 倍, 成为迄今为止正己 烷吸附量最高的 MOF 材料. 李静教授等进一步对 Zr-bptc 和 Zr-abtc 进行了穿透实验，以 $1 \mathrm{~mL} / \mathrm{min}$ 的干燥 氮气通入异构体混合液中, 鼓出的气流经过样品管，尾 端用气相色谱进行检测. 结果显示, Zr-bptc 和 $5 \AA$ 分子 笁都能将正己烷从 3-甲基戊烷和 2,3-二甲基丁烷中耖分 出来, 但由于 Zr-bptc 对正己烷高的吸附量, 其保留时间 (a)

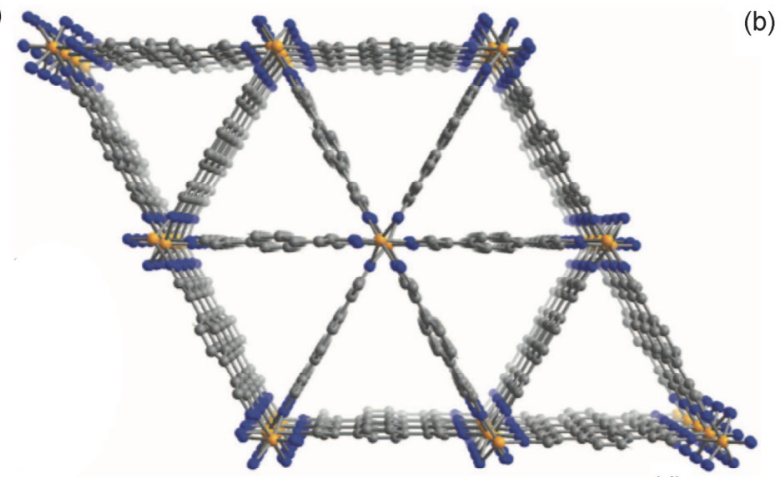

(c)

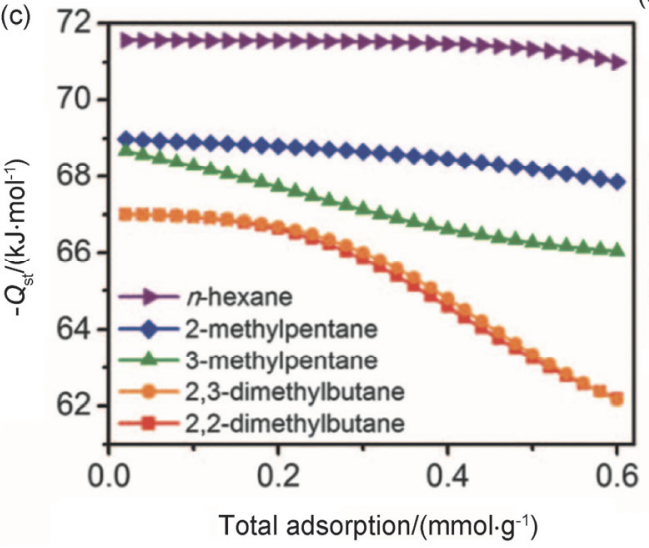

(b)
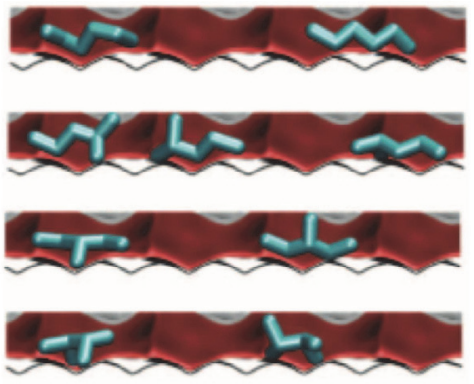

(d)

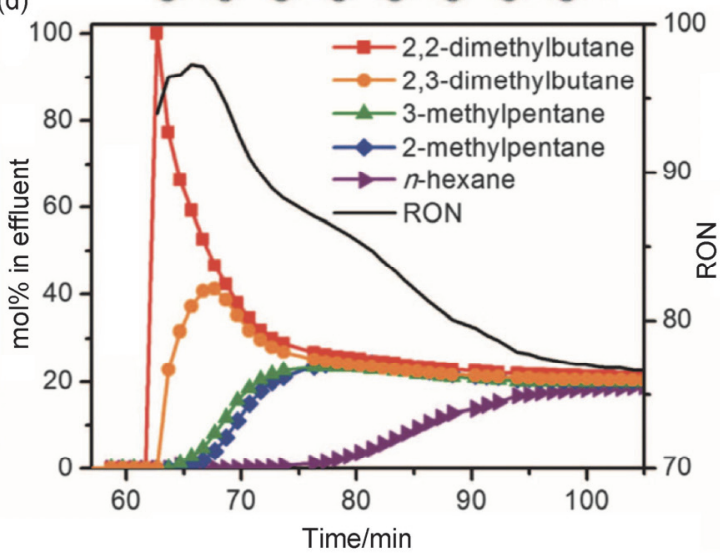

图 7 (a) $\mathrm{Fe}_{2}(\mathrm{BDP})_{3}$ 孔道结构示意图; (b) 己烷异构体在孔道中的构型偏倚蒙特卡洛(CBMC)模拟图; (c) $\mathrm{Fe}_{2}(\mathrm{BDP})_{3}$ 对已烷异构体的吸附焓曲线; (d) $\mathrm{Fe}_{2}(\mathrm{BDP})_{3}$ 对已烷异构体的穿透曲线. (改编自文献[26])

Figure 7 (a) Schematic diagram of the channel structure of $\mathrm{Fe}_{2}(\mathrm{BDP})_{3}$. (b) Illustration of hexane isomers in the channel in configurational-bias Monte Carlo $(\mathrm{CBMC})$ simulations. (c) Curves of isosteric heats of adsorption $\left(Q_{\mathrm{st}}\right)$. (d) Breakthrough curves for hexane isomers over $\mathrm{Fe}_{2}(\mathrm{BDP})_{3}$. $(\mathrm{Adapted}$ from the literature [26]) 
(a)

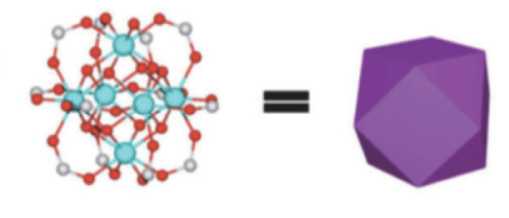

$\mathrm{Zr}_{6}\left(\mu_{3}-\mathrm{O}\right)_{4}\left(\mu_{3}-\mathrm{OH}\right)_{4}(\mathrm{COO})_{12}$
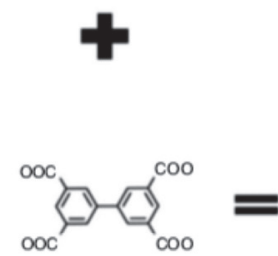

bptc
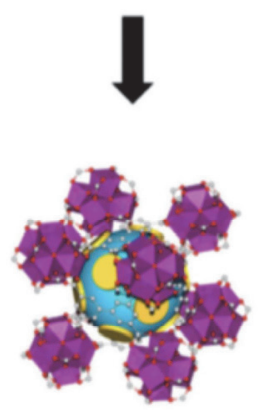

12-connected
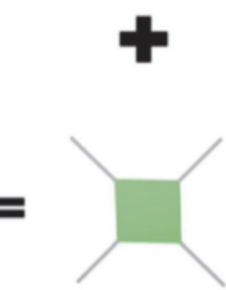

(c)

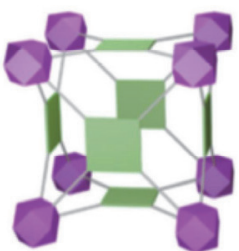

(b)
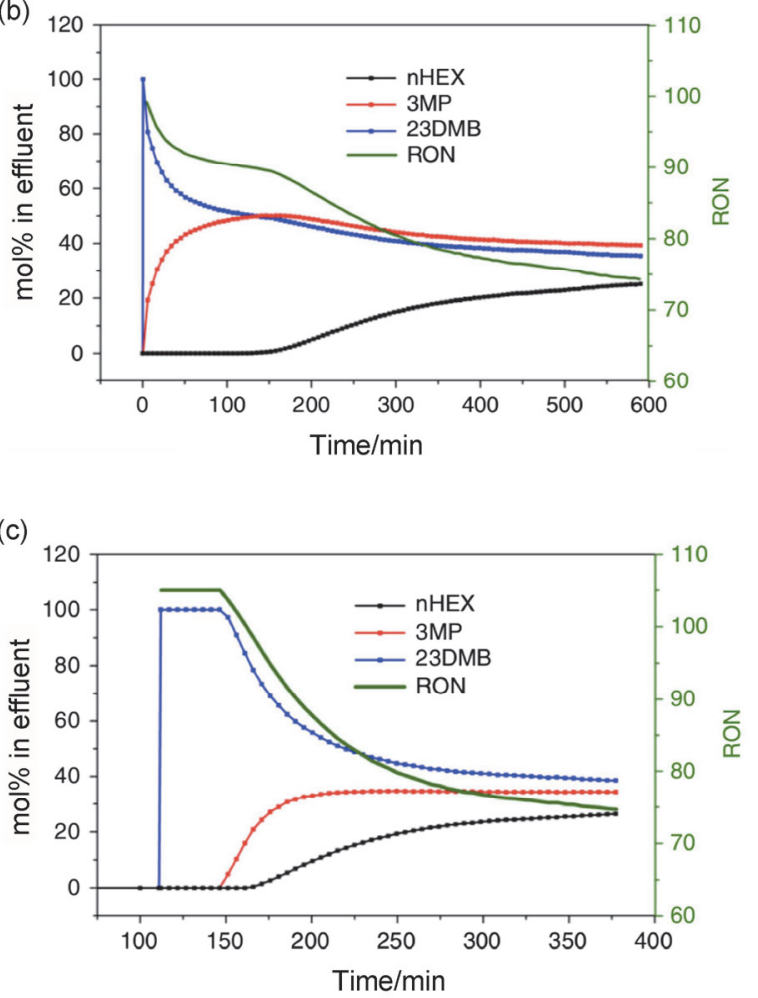

图 8 (a) Zr-bptc 结构单元及晶体结构示意图; (b) Zr-bptc 对正己烷、3-甲基戊烷和 2,3-二甲基丁烷的穿透曲线; (c) Zr-abtc 对正己烷、3-甲基戊烷 和 2,3-二甲基丁烷的穿透曲线. (改编自文献[29])

Figure 8 (a) Schematic diagram of unit and crystal structure of Zr-bptc; (b) Breakthrough curves for $n$-hexane/3-methylpentane/2,3-dimethylbutane (nHEX/3MP/23DMB) over Zr-bptc; (c) Breakthrough curves for nHEX/3MP/23DMB over Zr-abtc. (Adapted from the literature [29])

是 $5 \AA$ 分子筛的两倍(图 $8 b$ ). 值得一提的是, $\mathrm{Zr}$-abtc 除 了能有效分离正己烷之外, 还能实现 3-甲基戊烷和 2,3二甲基丁烷的篮分，进一步提高混合物的 RON 值(图 $8 \mathrm{c})$. 同年, 李静课题组 ${ }^{[30]}$ 还根据分子篮分效应, 选用微 孔 MOF 材料 $\mathrm{Ca}\left(\mathrm{H}_{2} \mathrm{tcpb}\right)$, 成功将单取代和双取代已烷 异构体分离. 更重要的是, 借助不同温度下己烷异构体 的吸附量差异，可利用温度程序设计，将线性、单取代 和双取代的三元混合物完全分离成纯组分.

\section{4 总结和展望}

轻烃分子的分离是石油化工领域中最重要的分离 过程之一, 相较于传统的精馏分离方式, 吸附式分离有 望实现高效、低能耗、低成本的分离, 其核心是吸附剂 材料. MOF 材料由于具有高比表面积, 易于调控的孔道 尺寸、形状和孔道环境, 丰富的官能团, 在分离纯化轻 烃分子中表现出巨大潜力. 根据目标分子的尺寸, 精准 调控 MOF 孔道尺寸和形状, 可通过尺寸篮分实现高选 择性分离. 此外, 选择含有特定官能团的 MOFs 或是通 过合成后修饰的方法引入, 以此调控待分离组分和骨架 间的相互作用, 也可提高材料的选择性. 除此之外, 还 可借助 MOFs 中的不饱和金属位点以及柔性的骨架结构 来实现高效分离.
随着 MOF 吸附剂材料的不断开发, 在评估分离性 能时, 评估标准和方式要尽量接近工业分离条件. 首先, 仅根据单组分的静态吸附曲线去分析材料分离性能是 远远不够的，还应考虑动力学过程以及多组分时偏离理 想状态的情况. 其次, 应同时关注材料的选择性和吸附 容量, 可以通过多组分动态穿透曲线获得这些关键信 息. 最后, 在测试过程中的各项参数如各组分的比例、 温度、压力等的调控应考虑应用背景, 以及实际应用中 的杂质气体或者水汽的干扰.

面向实际的分离场景，除了分离性能，对于吸附剂 材料的稳定性、加工性、成本都有一定要求, MOF 材料 在走向工业化的过程中仍然面临着许多挑战. 首先, 具 有优异分离性能的 MOF 材料的大规模生产存在巨大困 难. 合成 MOFs 的原料, 尤其是有机单体价格昂贵, 并 且 MOFs 的制备和纯化过程繁琐，阻碍了其工业化生产. 尽管目前可通过电解法、固相合成法等手段大批量制备 MOF 材料, 但是可合成的材料种类十分有限. 因此, 仍 需大力开发高性能 MOF 吸附剂的批量合成策略以及纯 化方法，拓展可生产 MOF 的种类，降低制备成本. 其 次, MOFs 造粒成型工艺仍需开发. MOFs 通常以粉末形 态合成得到，在实际分离过程中可能会堵塞管路或是随 气流扩散, 引起安全问题、材料损失等, 目前的造粒成 
型手段多是通过高压机械造粒或加入交联剂辅助成型, 前者可能会造成骨架的坍塌, 而后一种方式可能会堵塞 孔道, 均会对其分离性能造成严重影响, 因此在保证其 结构和孔道完整性的基础上发展造粒成型工艺十分重 要. 再次, 考虑到工业分离过程中的严苛条件, 应进一 步提高 MOFs 的化学稳定性和热稳定性, 以保证在高温 和腐蚀性杂质气体存在时材料的分离性能. 本课题组提 出 MOFs 孔内原位聚合策略, 利用疏水性高分子对孔道 进行二次分割, 不仅可提高气体的选择性, 还大大提高 了材料的水稳定性 ${ }^{[31]}$. 除此之外, MOFs 的使用周期和 循环稳定性也是工业分离应用中需要考虑的重要因素 之一, 因此不仅要保证材料在分离过程中的稳定性, 还 应考虑在活化条件下(如高温、高真空等)MOFs 结构和 性能的保持, 以实现 MOF 吸附剂材料的循环使用. 最 后, MOFs 的分离机理和构效关系仍需深入研究. 可通 过原位表征手段, 如冷冻电镜、同步辐射光源衍射等明 确吸附位点, 结合分子动力学模拟研究目标分子在孔道 内的扩散行为, 阐明构效关系, 在此基础上优化分子设 计, 提高分离性能.

本综述介绍了 $\mathrm{MOF}$ 材料在 $\mathrm{C}_{4} \sim \mathrm{C}_{6}$ 碳氢化合物分离 中的研究进展, 分析了 MOF 材料的孔道尺寸、形状、 框架结构和分离选择性、吸附容量之间的关系. 在此基 础上, 总结了 MOFs 吸附剂材料在实现工业化进程中面 临的挑战和未来的发展方向, 为开发高性能的 MOF 分 离材料提供了研究思路.

\section{作者简介}

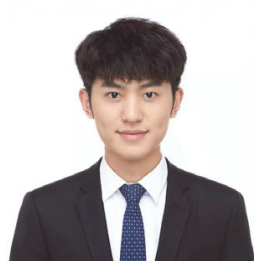

郭振涁, 北京理工大学化学与化工学院, 2017 级在读硕士 研究生(化学专业), 本科毕业于青岛科技大学(应用化学专业), 现主要从事晶态多孔材料的合成与应用研究.

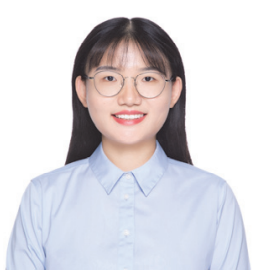

张媛媛, 北京理工大学前沿交叉科学研究院, 预聘助理 教授. 2013 年 2019 年在北京理工大学化学与化工学院获得 博士学位, 2017 年 2018 年在美国西北大学化学系访问学习 (联合培养博士), 2019 年就职于北京理工大学前沿交叉科学研 究院. 主要从事金属有机框架(MOF)、共价有机框架(COF)等 功能多孔材料的可控制备和柔性加工, 及其在分离、催化、智 能响应等领域的应用研究.

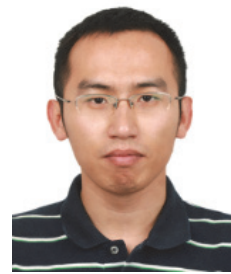

冯霄, 北京理工大学博士生导师, 化学与化工学院教授, 国家自然科学基金优秀青年科学基金获得者. 分别于 2008 年 和 2013 年于北京理工大学材料学院取得本科与博士学位, 攻 读博士期间以联合培养博士研究生身份留学于日本国家自然 科学研究机构——分子科学研究所. 2013 年就职于北京理工 大学化学与化工学院. 主要从事关于共价有机框架材料等晶 态多孔材料的构效关系研究以及膜分离相关领域应用研究.

\section{References}

[1] Bender, M. ChemBioEng Rev. 2014, 1, 136.

[2] Gehre, M.; Guo, Z.; Rothenberg, G.; Tanase, S. ChemSusChem 2017, $10,3947$.

[3] Ed.: Myers, R. A. Handbook of Petroleum Refining Processes, McGraw-Hill, New York, 2004.

[4] Greensfelder, B. S.; Voge, H. H. Ind. Eng. Chem. Res. 1945, 37, 514.

[5] Li, J.-R.; Kuppler, R. J.; Zhou, H.-C. Chem. Soc. Rev. 2009, 38, 1477.

[6] Tijsebaert, B.; Varszegi, C.; Gies, H.; Xiao, F. S.; Bao, X.; Tatsumi, T.; Muller, U.; De Vos, D. Chem. Commun. 2008, 2480.

[7] (a) Yaghi, O. M.; O’Keeffe, M.; Ockwig, N. W.; Chae, H. K.; Eddaoudi, M.; Kim, J. Science 2005, 310, 1166. (b) Kitagawa, S.; Kitaura, R.; Noro, S. Angew. Chem., Int. Ed. 2004, 43, 2334. (c) Ferey, G. Chem. Soc. Rev. 2008, 37, 191. (d) Farha, O. K.; Hupp, J. T. Acc. Chem. Res. 2010, 43, 1166. (e) Eddaoudi, M.; Li, H.; Yaghi, O. M. J. Am. Chem. Soc. 2000, 122, 1391. (f) Farha, O. K.; Eryazici, I.; Jeong, N. C.; Hauser, B. G.; Wilmer, C. E.; Sarjeant, A. A.; Snurr, R. Q.; Nguyen, S. T.; Yazaydın, A. Ö.; Hupp, J. T. J. Am. Chem. Soc. 2012, 134, 15016.

[8] (a) Sumida, K.; Rogow, D. L.; Mason, J. A.; McDonald, T. M.; Bloch, E. D.; Herm, Z. R.; Bae, T.-H.; Long, J. R. Chem. Rev. 2012, 112, 724. (b) Makal, T. A.; Li, J. R.; Lu, W.; Zhou, H. C. Chem. Soc. Rev. 2012, 41, 7761. (c) Murray, L. J.; Dinca, M.; Long, J. R. Chem. Soc. Rev. 2009, 38, 1294. (d) Zhao, X.; Wang, Y.; Li, D. S.; Bu, X.; Feng, P. Adv. Mater. 2018, 30, 1705189. (e) Adil, K.; Belmabkhout, Y.; Pillai, R. S.; Cadiau, A.; Bhatt, P. M.; Assen, A. H.; Maurin, G.; Eddaoudi, M. Chem. Soc. Rev. 2017, 46, 3402. (f) Van de Voorde, B.; Denayer, J.; De Vos, D. Chem. Soc. Rev. 2014, 43, 5766. (g) Holst, J. R.; Trewin, A.; Cooper, A. I. Nat. Chem. 2010, 2, 915. (h) Farha, O. K.; Yazaydin, A. Ö.; Eryazici, I.; Malliakas, C. D.; Hauser, B. G.; Kanatzidis, M. G.; Nguyen, S. T.; Snurr, R. Q.; Hupp, J. T. Nat. Chem. 2010, 2, 944. (i) Eddaoudi, M.; Kim, J.; Rosi, N.; Vodak, D.; Wachter, J.; O'Keeffe, M.; Yaghi, O. M. Science 2002, 295, 469. (j) Liu, Y.; Xia, X.-X.; Tan, Y.-Y.; Li, S. Acta Chim. Sinica 2020, 78, 250 (in Chinese). (刘洋, 夏㴋㴋, 谭媛元, 李松, 化学学报, 2020, 78, 250.) (k) Chen, Z.-Y.; Liu, J.-W.; Cui, H.; Zhang, L.; Su, C.-Y. Acta Chim. Sinica 2019, 77, 242 (in Chinese). (陈之尧, 刘捷威, 崔 浩, 张利, 苏成勇, 化学学报, 2019, 77, 242.) (1) Bian, L.; Li, W.; Wei, Z.-Z.; Liu, X.-W.; Li, S. Acta Chim. Sinica 2018, 76, 303 (in Chinese). (市磊, 李炜, 魏振振, 刘晓威, 李松, 化学学报, 2018, 76, 303.) (m) Yang, W.-Y.; Liang, H.; Qiao, Z.-W. Acta Chim. Sinica 2018, 76, 785 (in Chinese). (杨文远, 梁红, 乔智威, 化学学报, 2018, 76, 785.)

[9] (a) Yi, F.-Y.; Chen, D.; Wu, M.-K.; Han, L.; Jiang, H. L. ChemPlusChem 2016, 81, 675. (b) Kempahanumakkagari, S.; Kumar, V.; Samaddar, P.; Kumar, P.; Ramakrishnappa, T.; Kim, K.-H. Biotechnol. Adv. 2018, 36, 467. (c) Dolgopolova, E. A.; Rice, A. M.; Martin, C. R.; Shustova, N. B. Chem. Soc. Rev. 2018, 47, 4710. (d) Furukawa, H.; Cordova, K. E.; O'Keeffe, M.; Yaghi, O. M. Science 2013, 341, 1230444. (e) Hu, Z.; Deibert, B. J.; Li, J. Chem. Soc. Rev. 2014, 43, 5815. (f) Sun, Y.-H.; Qi, Y.-X.; Shen, Y.; Jing, C.-J.; Chen, 
X.-X.; Wang, X.-X. Acta Chim. Sinica 2020, 78, 147 (in Chinese). (孙延慧, 齐有啸, 申优, 井翠洁, 陈笑笑, 王新星, 化学学报, 2020, 78, 147.) (g) Pang, C.-M.; Luo, S.-H.; Hao, Z.-F.; Gao, J.; Huang, Z.-H.; Yu, J.-H.; Yu, S.-M.; Wang, Z.-Y. Chin. J. Org. Chem. 2018, 38, 2606 (in Chinese). (庞楚明, 罗时荷, 郝志峰, 高健, 黄 召吴，余家海，余思敏，汪朝阳，有机化学， 2018，38，2606.) (h) Shi, Y.-X.; Zhang, W.-H.; Abrahams, B. F.; Braunstein, P.; Lang, J.-P. Angew. Chem., Int. Ed. 2019, 58, 9453.

[10] (a) Jiao, L.; Wang, Y.; Jiang, H. L.; Xu, Q. Adv. Mater. 2018, 30, 1703663. (b) Cui, W.-G.; Zhang, G.-Y.; Hu, T.-L.; Bu, X.-H. Coord. Chem. Rev. 2019, 387, 79. (c) Diercks, C. S.; Liu, Y.; Cordova, K. E.; Yaghi, O. M. Nat. Mater. 2018, 17, 301. (d) Lv, X. L.; Wang, K.; Wang, B.; Su, J.; Zou, X.; Xie, Y.; Li, J. R.; Zhou, H. C. J. Am. Chem. Soc. 2017, 139, 211. (e) Lee, J.; Farha, O. K.; Roberts, J.; Scheidt, K. A.; Nguyen, S. T.; Hupp, J. T. Chem. Soc. Rev. 2009, 38, 1450. (f) Wu, Z.-M.; Shi, Y.; Li, C.-Y.; Niu, D.-Y.; Chu, Q.; Xiong, W.; Li, X.-Y. Acta Chim. Sinica 2019, 77, 758 (in Chinese). (武卓 敏, 石勇, 李春艳, 牛丹阳, 楚奇, 熊巍, 李新勇, 化学学报, 2019, 77, 758.) (g) Xu, H.; Zhang, M.-Y.; Huang, X.; Shi, D.-B. Chin. J. Org. Chem. 2018, 38, 832 (in Chinese). (徐缓, 张茂元, 黄 香, 史大斌, 有机化学, 2018, 38, 832.) (h) Guo, X.-L.; Chen, X.; Su, D.-S.; Liang, C.-H. Acta Chim. Sinica 2018, 76, 22 (in Chinese). (郭小玲，陈霄，苏党生，梁长海，化学学报，2018，76，22.) (i) Huang, G.; Chen, Y.-Z.; Jiang, H. L. Acta Chim. Sinica 2016, 74, 113 (in Chinese). (黄刚, 陈玉贞, 江海龙, 化学学报, 2016, 74, 113.) (j) Jiao, L.; Jiang, H. L. Chem 2019, 5, 786. (k) Xiao, J.-D.; Jiang, H. L. Acc. Chem. Res. 2019, 52, 356. (1) Li, F. L.; Wang, P. T.; Huang, X. Q.; Young, D. J.; Wang, H. F.; Braunstein, P.; Lang, J. P. Angew. Chem., Int. Ed. 2019, 58, 7051. (m) Hu, F.-L.; Mi, Y.; Zhu, C.; Abrahams, B. F.; Braunstein, P.; Lang, J. P. Angew. Chem., Int. Ed. 2018, 57, 12696.

[11] (a) Horcajada, P.; Gref, R.; Baati, T.; Allan, P. K.; Maurin, G.; Couvreur, P.; Férey, G.; Morris, R. E.; Serre, C. Chem. Rev. 2012, 112, 1232. (b) Zhou, H. C.; Long, J. R.; Yaghi, O. M. Chem. Rev. 2012 , 112, 673. (c) Giménez, M.-M.; Hidalgo, T.; Serre, C.; Horcajada, P. Coord. Chem. Rev. 2016, 307, 342. (d) Zeng, J.-Y.; Wang, X.-S.; Zhang, X.-Z.; Zhuo, R.-X. Acta Chim. Sinica 2019, 77, 1156 (in Chinese). (曾锦跃, 王小双, 张先正, 卓仁禧, 化学学报, 2019, $77,1156$.

[12] (a) Cui, W. G.; Hu, T. L.; Bu, X. H. Adv. Mater. 2019, 32, 1806445. (b) Li, J. R.; Kuppler, R. J.; Zhou, H. C. Chem. Soc. Rev. 2009, 38, 1477.

[13] Sircar, S.; Mohr, R.; Ristic, C.; Rao, M. B. J. Phys. Chem. B 1999, 103,6539 .
[14] Hartmann, M.; Kunz, S.; Himsl, D.; Tangermann, O.; Ernst, S.; Wagener, A. Langmuir 2008, 24, 8634.

[15] Schoonheydt, R. A.; Weckhuysen, B. M. Phys. Chem. Chem. Phys. 2009, 11, 2794

[16] Barnett, B. R.; Parker, S. T.; Paley, M. V.; Gonzalez, M. I.; Biggins, N.; Oktawiec, J.; Long, J. R. J. Am. Chem. Soc. 2019, 141, 18325.

[17] Jiao, J.; Liu, H.; Bai, D.; He, Y. Inorg. Chem. 2016, 55, 3974.

[18] Kim, H.; Park, J.; Jung, Y. Phys. Chem. Chem. Phys. 2013, 15, 19644.

[19] Jiao, J.; Liu, H.; Bai, D.; He, Y. Inorg. Chem. 2016, 55, 3974

[20] Zhang, Z.; Yang, Q.; Cui, X.; Yang, L.; Bao, Z.; Ren, Q.; Xing, H. Angew. Chem., Int. Ed. 2017, 56, 16282.

[21] Cui, J.; Zhang, Z.; Tan, B.; Zhang, Y.; Wang, P.; Cui, X.; Xing, H. Chem. Asian. J. 2019, 14, 3572.

[22] Lange, M.; Kobalz, M.; Bergmann, J.; Lässig, D.; Lincke, J.; Möllmer, J.; Möller, A.; Hofmann, J.; Krautscheid, H.; Staudt, R.; Gläser, R. J. Mater. Chem. A 2014, 2, 8075.

[23] Kishida, K.; Okumura, Y.; Watanabe, Y.; Mukoyoshi, M.; Bracco, S.; Comotti, A.; Sozzani, P.; Horike, S.; Kitagawa, S. Angew. Chem., Int. Ed. 2016, 55, 13784.

[24] Liao, P.-Q.; Huang, N.-Y.; Zhang, W.-X.; Zhang, J.-P.; Chen, X.-M. Science 2017, 356, 1193.

[25] Chen, B.; Liang, C.; Yang, J.; Contreras, D. S.; Clancy, Y. L.; Lobkovsky, E. B.; Yaghi, O. M.; Dai, S. Angew. Chem., Int. Ed. 2006, 45, 1390.

[26] Herm, Z. R.; Wiers, B. M.; Mason, J. A.; Baten, J. M.; Hudson, M. R.; Zajdel, P.; Brown, C. M.; Masciocchi, N.; Krishna, R.; Long, J. R. Science 2013, 340, 960.

[27] Mendes, P. A. P.; Horcajada, P.; Rives, S.; Ren, H.; Rodrigues, A. E.; Devic, T.; Magnier, E.; Trens, P.; Jobic, H.; Ollivier, J.; Maurin, G.; Serre, C.; Silva, J. A. C. Adv. Funct. Mater. 2014, 24, 7666.

[28] Assen, A. H.; Belmabkhout, Y.; Adil, K.; Bhatt, P. M.; Xue, D. X.; Jiang, H.; Eddaoudi, M. Angew. Chem., Int. Ed. 2015, 54, 14353.

[29] Wang, H.; Dong, X.; Lin, J.; Teat, S. J.; Jensen, S.; Cure, J.; Alexandrov, E. V.; Xia, Q.; Tan, K.; Wang, Q.; Olson, D. H.; Proserpio, D. M.; Chabal, Y. J.; Thonhauser, T.; Sun, J.; Han, Y.; Li, J. Nat. Commun. 2018, 9, 1745.

[30] Wang, H.; Dong, X.; Velasco, E.; Olson, D. H.; Han, Y.; Li, J. Energy Environ. Sci. 2018, 11, 1226.

[31] Ding, N.; Li, H.-W.; Wang, Q.-Y.; Wang, S.; Ma, L.; Zhou, J.-W.; Wang, B. J. Am. Chem. Soc. 2016, 138, 10100. 